\title{
Mapping the caregiving process in pediatric asthma: Parental burden, acceptance and denial coping strategies and quality of life
}

\author{
Neuza Silva a ${ }^{-}$Carla Crespo ${ }^{b}$ Carlos Carona a,c $\bullet$ Maria Cristina Canavarro a \\ a Faculty of Psychology and Education Sciences, University of Coimbra, Coimbra, Portugal; \\ b Faculty of Psychology, University of Lisbon, Lisbon, Portugal; \\ c Coimbra Cerebral Palsy Association, Coimbra, Portugal
}

\begin{abstract}
Introduction. Based on a multi-dimensional model of the caregiving process, the main goal of this study was to examine the direct and indirect links, via acceptance and denial coping, between the caregiving burden and the quality of life (QoL) in parents of children with asthma. Method. The sample was composed of 182 parents of a child/adolescent between 8 and 18 years of age with a clinical diagnosis of asthma. Data were obtained via self-report questionnaires assessing the caregiving burden, acceptance and denial coping strategies and QoL. Results. Results from structural equation modelling indicated a good fit for the mediation model, which explained $30 \%$ of the variability of the parents' QoL. Higher levels of caregiving burden were negatively and indirectly associated with the parents' QoL, via less use of acceptance and greater use of denial coping strategies. Multi-group analyses ascertained the invariance of these links across the children's asthma severity, age and socio-economic groups. Conclusion. These findings emphasize acceptance and denial as important coping mechanisms in the caregiving process. Thus, broad-spectrum familycentered interventions in pediatric asthma settings can target the development of the parents' coping tendencies characterized by greater acceptance and less denial as a way of reappraising caregiving demands as less burdensome and improving their QoL.
\end{abstract}

\section{Keywords}

Acceptance/ denial coping strategies $•$ Caregiving burden - Multi-dimensional model of caregiving process and caregiver burden $\bullet$ Parental quality of life $\bullet$ Pediatric asthma

Silva, N., Crespo, C., Carona, C., \& Canavarro, M. C. (20I5). Mapping the caregiving process in paediatric asthma: Parental burden, acceptance and denial coping strategies and quality of life. Psychology \& Health, 30(8), 949-968. doi:I0.1080/08870446.20I5.100798I 


\section{Introduction}

Pediatric asthma has been suggested as a potentially stressful context for parental caregiving. The presence of this chronic condition implies the rearrangement of family routines to incorporate its management, such as avoiding environmental and emotional triggers of asthma exacerbations, monitoring symptoms and medication intake, and coordinating family and work responsibilities (Fiese, Winter, Anbar, Howell, \& Poltrock, 2008; Gates \& Akabas, 20I2). These caregiving demands can become burdensome and may have a deleterious effect on the parents' quality of life (QoL) outcomes (Crespo, Carona, Silva, Canavarro, \& Dattilio, 20II; Fiese, Wamboldt, \& Anbar, 2005). In fact, impaired physical functioning has been consistently described for parents of pediatric asthma patients when compared with the parents of healthy children (Gau et al., 20I0; Hatzmann, Heymans, Ferrer-i-Carbonell, van Praag, \& Grootenhuis, 2008; van Gent et al., 2007); conversely, research comparing the parents' psychological and social functioning has yielded heterogeneous findings across studies, as well as substantial within-study variability (Silva, Carona, Crespo, \& Canavarro, 2015). The variability in parental adaptation trajectories may be better explained not only by caregiving demands and perceived burden but also by a complex interplay between the risk factors and the resources, namely the coping processes (King, King, Rosenbaum, \& Goffin, 1999; Raina et al., 2004; Wallander et al., 1989). However, specific coping mechanisms remain empirically understudied in the pediatric asthma context. The examination of the mediating role of specific coping strategies on the associations between the caregiving burden and the parents' QoL may contribute to a better understanding of the caregiving process and to the identification of modifiable variables that could be targeted in psychosocial interventions aimed at promoting the adaptation of these parents.

A multi-dimensional approach to the caregiving process and parental adaptation

Raina and colleagues (2004) have proposed a multi-dimensional model of the caregiving process aimed at mapping the complex associations between contextual variables (e.g., socioeconomic status [SES]), child characteristics (e.g., disease severity), psychosocial stressors (e.g., caregiving burden), intrapersonal factors (e.g., perception of caregiving mastery), social-ecological factors (e.g., family functioning and social support), stress processing mechanisms (e.g., cognitive appraisal and coping strategies) and adaptation outcomes. Within this conceptual framework, the caregiving burden has been typically addressed as a risk factor for poor parental adaptation outcomes. Even if providing daily care to children is a normative assignment of parenting, the increased responsibilities and limitations experienced by parents caring for a child with a chronic condition may elicit caregiving stress related to the parent-child dyadic relationship (relationship burden), instrumental activities and consequent time constraints (objective burden), and emotional 
stress and anxiety (subjective burden; Savundranayagam, Montgomery, \& Kosloski, 20I I). Higher levels of caregiving burden have been associated with psychological maladjustment in the caregivers of children with chronic medical conditions (Canning, Harris, \& Kelleher, 1996) and with impaired QoL in the parents of children with asthma (Fiese et al., 2005). However, these pathways have not always emerged directly, but were mediated by social-ecological factors, such as family functioning (Crespo et al., 20II) and social support (Carona, Crespo, \& Canavarro, 20I3).

The disease severity, the child's age and family SES are also relevant risk factors, which can operate at different levels of the caregiving process. For example, disease severity has been associated with a greater caregiving burden in parents caring for a child with a chronic condition (Canning et al., 1996) and with decreased QoL in parents in the particular context of pediatric asthma (Everhart, Fiese, \& Smyth, 2008; Halterman et al., 2004). The effects of the child's age on the caregiving burden and QoL outcomes have also been tested, but studies have yielded divergent results, depending on the child's health condition. For example, higher levels of objective burden and lower QoL were described for parents of adolescents compared to parents of younger children with cerebral palsy (Carona, Pereira, Moreira, Silva, \& Canavarro, 2013). However, the inverse pattern of age-group differences emerged in non-clinical groups (Carona, Pereira et al., 2013) and mixed samples of parents of pediatric patients with chronic health conditions and disabilities (Floyd \& Gallagher, 1997). Considering that pediatric asthma does not seriously threaten the development of autonomy in adolescence (as in the case of cerebral palsy), it can be hypothesized that younger children would require greater assistance in the activities of daily living and asthma management, which may result in an increased caregiving burden and a lower parental QoL. Furthermore, providing treatments and additional care required by a child with asthma may increase the financial burden (Gates \& Akabas, 2012), and thus family SES has been acknowledged as a significant risk factor for QoL impairments in the caregivers of children with asthma (Everhart et al., 2008).

Consistent with the stress and coping theory, which advocates that adaptation outcomes are determined by an individual's appraisal of stressors and resources (Lazarus \& Folkman, 1984), the caregiving process model considers the stress management strategies as constraining resources with regard to the caregiver's adaptation outcomes (Raina et al., 2004). Coping, defined as "the cognitive and behavioral efforts made to master, tolerate, or reduce external and internal demands and conflicts among them" (Folkman \& Lazarus, 1980, p. 223), has been referred to as a dynamic process with a protective function, removing or changing the stressor through the reappraisal of its meaning (i.e., problem-focused coping) or by the regulation of subsequent emotions (i.e., emotion-focused coping; Pearlin \& Schooler, 1978). The use of specific coping strategies would be mainly determined by the individual's appraisal of the stressful situation (primary appraisal) and the available resources (secondary appraisal; Lazarus \& Folkman, 1984), and could influence the subsequent adaptation outcomes or even enhance personal growth (Blount et al., 2008). Some authors have advocated that 
when the stressful situation is appraised as persisting over time and unlikely to undergo beneficial changes, as in many cases of chronic health conditions, emotion-focused coping strategies aimed at managing the consequent emotional distress are preferred (Carver, Scheier, \& Weintraub, 1989; Folkman \& Lazarus, 1980).

Acceptance and denial as coping strategies in the caregiving process

One specific emotion-focused coping strategy is accepting the reality of the stressful situation, which was argued by Carver and colleagues (1989) to be a functional coping response, because it would motivate active attempts to address the stressor. In the context of family caregiving, acceptance may reflect an attempt to make sense of the child's health condition and a reconciliation of the caregiving demands with the caregiver's goals, values and beliefs, as well as a sense of control over emotional reactions (Pakenham, 2008). The coping response of acceptance has been related to a greater life satisfaction and positive affect, and less anxiety and depression in the caregivers of adult patients (Pakenham, 2008). The third wave of cognitive-behavioral therapies (e.g., Acceptance and Commitment Therapy [ACT], Hayes, 2004; Mindfulness-Based Stress Reduction [MBSR], Kabat-Zinn, 1990) relies specifically on strategies such as mindfulness, acceptance or cognitive defusion to change the meaning of the stressful events and to develop more flexible and effective coping repertoires (Hayes, 2004; Larson, 2010). There is scarce but promising evidence that this intervention approach may be effective in reducing parenting stress, depression and negative affect, and in improving psychological well-being and QoL outcomes in the parents of children with chronic conditions or disabilities (e.g., Bazzano et al., 20I5; Benn, Akiva, Arel, \& Roeser, 2012; Minor, Carlson, Mackenzie, Zernicke, \& Jones, 2006).

In opposition, denial has been considered a particular form of cognitive avoidance (Moos \& Schaefer, 1993), which is characterized by attempts to deny or minimize the importance of a stressful event or its consequences, and by behavioral efforts to ignore the stressor (Carver et al., 1989). Although denial is considered a dynamic process that may have a protective function depending on the context and the time in which it operates (e.g., following the diagnosis of a chronic illness; Livneh, 2009), avoidant strategies have been systematically associated with poorer psychological adjustment to a physical illness (Roesch \& Weiner, 200I). In parents caring for a child with cerebral palsy, experiential avoidance was positively related to the parenting burden and symptoms of depression, anxiety and stress (Whittingham, Wee, Sanders, \& Boyd, 2013). Moreover, disengagement coping strategies, including cognitive avoidance, were identified as mediators of the associations between the caregiving burden and anxiety and depression in the caregivers of adult patients with Alzheimer's disease (García-Alberca et al., 2012).

It is also important to note that although acceptance and denial have been conceptualized in a somewhat opposite way, they are likely co-occurring responses to different aspects of the stressful 
situation (Carver et al., 1989). For instance, a parent may tend to accept the disruption of daily routines implied by caregiving, and yet being prone to deny negative emotional states aroused by the child's physical impairment and/or treatment procedures. Hence, acceptance and denial responses and their links to parents' QoL must be examined separately, instead of being considered as opposite and mutually exclusive sides of the same coping strategy.

The present study: Aims and hypotheses

Within the broader concept of "adaptation", the parents' QoL was defined as the "individuals' perceptions of their position in life in the context of the culture and value systems in which they live and in relation to their goals, expectations, standards and concerns" (The WHOQOL Group, 1998, p. 55I) and considered as an overall indicator of their physical, psychological and social well-being. In the context of family caregiving for a child with asthma, the following objectives were outlined for the present study: (I) to characterize the parents' caregiving burden, coping tendencies and QoL in different clinical (intermittent vs. persistent asthma) and socioeconomic groups (low vs. medium/high SES), and at different developmental periods (childhood vs. adolescence); (2) to examine the direct and indirect links, via acceptance and denial as dispositional coping strategies, between the caregiving burden and the parents' QoL; and (3) to attest if the mediation model was moderated by asthma severity, child's age and family SES. Following the recommendations of Raina and colleagues (2004), we used advanced statistical techniques (structural equation modelling [SEM]) that allowed the operationalization of the caregiving burden and QoL as multi-dimensional constructs and the examination of the complex mechanisms (i.e., direct and indirect pathways) underpinning the caregiving process and the parents' adaptation outcomes.

Accordingly, the following theoretically driven hypotheses were formulated. First, we expected higher levels of caregiving burden and lower QoL in the parents caring for a child with persistent asthma, the parents of younger children and the parents with low SES when compared with the parents caring for a child with intermittent asthma, the parents of adolescents and the parents with medium/high SES. Second, we predicted that higher levels of caregiving burden would be associated with lower parental QoL and that this negative association would be mediated by coping strategies, such as a reduced use of acceptance and a greater use of denial. We also tested the invariance of the mediation model across the asthma severity, the children's age and the socioeconomic groups, but, given the exploratory nature of these analyses, no predictions were made in this regard. 


\section{Method}

\section{Participants and procedure}

The present study is part of a broader research project, which was approved by the ethics committees and/or direction boards of three Portuguese public hospitals (Coimbra University Hospitals, Coimbra Pediatric Hospital, and Leiria Santo André Hospital). Parents were enrolled at the Pediatric and Immunoallergology outpatient services of the aforementioned hospitals between September 2010 and February 2012 if they had a child who met the following criteria: (I) between 8 and 18 years of age; (2) diagnosis of asthma for at least one year that was established by a physician according to the International Classification of Diseases system (ICD-I0); and (3) absence of comorbidities with other chronic health conditions or severe psychiatric disorders, except for comorbid allergic diseases (e.g., allergic rhinitis, atopic dermatitis/ eczema, and hypersensitivity to inhalant or food allergens). In addition, an inclusion criterion for parents was considered: being the parent self-identified as currently assuming the role of primary caregiver for the child's asthma management.

The medical files of the children attending medical routine appointments in the period of data collection were screened by their physicians. Adopting a non-probabilistic convenience sampling method, the primary caregivers of the eligible children were invited to participate in the study. Detailed information about the study's aims and procedures was provided to all eligible participants, and informed consent was obtained from the parents who agreed to participate. The parents completed a set of self-report questionnaires in a room assigned for research purposes in the health institution that their child attended. A research assistant was available to provide occasional support to the participants with difficulties in reading or understanding item content.

\section{Measures}

\section{Caregiving burden}

The three burden subscales of the Revised Burden Measure (Montgomery \& Kosloski, 2006; Portuguese version: ongoing psychometric studies) were used to assess the extent to which various aspects of parents' lives had changed due to caregiving for their children with asthma ("As a result of assisting the care receiver, have the following aspects of your life changed?"). The Relationship burden subscale (five items) assesses the negative psychological state that directly derives from the caregiver's unique relationship with the care receiver, particularly as a result of demands for care and attention over and above the level that the caregiver perceives is warranted by the care receiver's condition (e.g., "Have your caregiving responsibilities caused conflicts with your 
relative?"). The Objective burden subscale (six items) assesses the negative psychological state that results from the interference of caregiving activities on the caregiver's free time (e.g., "Have your caregiving responsibilities kept you from recreational activities?"). The Subjective burden subscale (five items) refers to a generalized form of negative affect resulting from caregiving, but not as a direct consequence of any specific event, task or interaction (e.g., "Have your caregiving responsibilities created a feeling of hopelessness?"). The parents answered a total of 16 items using a five-point Likert scale, which ranged from I (not at all) to 5 (a great deal), with higher scores indicating greater levels of caregiving burden. For SEM, the three subscales were considered observed indicators, loading on a latent variable representing the multi-dimensional construct of caregiving burden. As displayed in Table 2, good reliability was found in the current sample for the three subscales and for the latent variable, resembling the Cronbach's $\alpha$ values described in the original study with a sample of spouses and adult children caregivers of persons with chronic illnesses ( $\alpha$ ranging from .85 to .93; Savundranayagam et al., 20I I).

\section{Acceptance and denial coping strategies}

The parents' use of acceptance and denial as dispositional coping strategies was evaluated with the respective subscales of the Brief-COPE (Carver, 1997; Portuguese version: Pais-Ribeiro \& Rodrigues, 2004). The Acceptance subscale is composed of two items that assess the parents' recognition and acceptance that the stressful event has occurred and is real ("I've been accepting the reality of the fact that it has happened."; "I've been learning to live with it."). The Denial subscale, which is also composed of two items, assesses the parents' cognitive and behavioral attempts to reject or ignore the actual existence of the stressful event ("I've been saying to myself 'this isn't real'."; "I've been refusing to believe that it has happened."). For both subscales, the answers were provided using a dispositional response format with four options, ranging from 0 (I usually don't do this at all) to 3 (I usually do this a lot), with higher scores indicating more frequent use of the coping strategy. For SEM, we considered acceptance and denial as latent variables, which were reflected in their respective two items as observed indicators. In the current sample, the Cronbach's $\alpha$ coefficients were .63 for the Acceptance subscale and .58 for the Denial subscale (composite reliability values for the latent variables are presented in Table 2). These reliability values exceeded those reported in the original studies of the Brief-COPE instrument $(\alpha=.57$ for Acceptance and .54 for Denial) and that were considered to be acceptable by the author (Carver, 1997).

\section{Quality of life}

The parents' QoL was assessed with the abbreviated version of the World Health Organization Quality of Life questionnaire (WHOQOL- BREF; The WHOQOL Group, 1998; Portuguese version: Vaz-Serra et al., 2006). This questionnaire includes 26 items clustered into a 
generic facet composed of two items (which were not used in the present study) and four QoL domains. The Physical domain assesses the facets of pain and discomfort, energy and fatigue, sleep and rest, dependence on medication, mobility, activities of daily living and working capacity (seven items; e.g., "Do you have enough energy for everyday life?”). The Psychological domain encompasses the facets of positive and negative feelings, self-esteem, thinking, learning, memory and concentration, body image and spirituality, religion and personal beliefs (six items; e.g., "How often do you have negative feelings such as blue mood, despair, anxiety, depression?"). The Social Relationships domain comprises the facets of personal relationships, sexual activity and social support (three items; e.g., "How satisfied are you with the support you get from your friends?"). The Environment domain evaluates the facets of physical safety and security, the home and physical environments, financial resources, access to health and social care, transport, opportunities for acquiring new information and skills, and recreation and leisure activity (eight items; e.g., "How healthy is your physical environment?"). The items were answered using a five-point Likert scale in terms of intensity (not at all to extremely), capacity (not at all to completely), frequency (never to always) or evaluation (very dissatisfied to very satisfied), with higher values indicating a better QoL. A latent variable representing the multi-dimensional construct of QoL, which comprised the four domains as observed indicators, was designed for the SEM. The cross-cultural psychometric study of the WHOQOL-BREF, involving 15 centers worldwide, attested the instrument's good internal consistency (Cronbach's $\alpha$ values ranging from .66 for Social Relationships domain to .84 for Physical domain; The WHOQOL Group, 1998). Similarly, good reliability was observed in the current sample for all four domains and for the latent variable (Table 2).

\section{Socio-demographic and clinical data}

The parents also completed a socio-demographic datasheet, which collected information on their age, gender, marital status, educational level and occupational status, as well as their children's age, gender and clinical information (e.g., use of medication, comorbidities with other health conditions). Using a classification system specifically developed for the Portuguese context and based on the educational level and current job of the primary caregiver (Simões, 1994), the family SES was classified into low (e.g., unqualified employees in construction or manufacturing without completing the $9^{\text {th }}$ grade of school education), medium (e.g., employees in bureaus or banks, nurses, social workers or teachers with intermediate or university courses) and high (e.g., senior officials of government, army, commerce or industry, physicians, magistrates or engineers with bachelor, master, doctorate or other post-graduate degrees).

Asthma severity was classified by the child's physician into four levels (intermittent, mild persistent, moderate persistent and severe persistent), according to the Global Initiative for Asthma [GINA] guidelines (2008). This classification was based on asthma clinical features before treatment, 
as recorded in the child's medical files, namely the level of symptoms, airflow limitation and lung function variability.

Statistical analyses

The statistical analyses were conducted with SPSS v.20.0 (SPSS Inc., Chicago, IL, USA). Except for socio-demographic and clinical variables, missing data, which were random and less than $5 \%$ of the values, were replaced with the individual mean score for each variable. Descriptive statistics were calculated for the socio-demographic, clinical and psychosocial variables. Due to the heterogeneous distribution of the asthma severity and the SES levels observed in our sample, for the group comparison analyses, these variables were dichotomized and dummy-coded (asthma severity: 0 - intermittent [ $n=95], I-$ persistent [mild, moderate or severe, $n=87]$; SES: 0 - low [ $n=108]$, $I$ - medium/high $[n=74]$ ). Multivariate analyses of covariance (MANCOVA) were performed to examine the main and interaction effects of the asthma severity (intermittent vs. persistent), the children's age (children aged 8-12 vs. adolescents aged 13-18) and the SES groups (low vs. medium/high) on the caregiving burden, the coping strategies and the parents' QoL. The remaining clinical and socio-demographic variables that were significantly associated with the main study variables were included as covariates. When multivariate effects were significant, univariate analyses were performed to examine which dimensions of the caregiving burden, coping and QoL significantly differed between the groups.

SEM was performed with Analysis of Moment Structures (AMOS Development Corporation, Meadville, PA). The method of estimation was the maximum likelihood, and the overall model fit was evaluated based on the chi-square statistic $\left(\chi^{2}\right)$ and on the main approximate goodness-of-fit indexes, namely, the comparative fit index (CFI), the root mean square error of approximation (RMSEA) and the standardized root mean squared residual (SRMR). A model was considered to have a good fit when $\chi^{2}$ was non-significant $(p>.05)$, the CFI $\geq .95$, the RMSEA $\leq .06(p>.05)$ and the SRMR $\leq .08$; an acceptable fit was defined by a CFI $\geq .90$ and a RMSEA $\leq .10$ (Browne \& Cudeck, 1993; Hu \& Bentler, 1999). To ensure the pertinence of multi-dimensional constructs (latent variables), we first examined the measurement model testing the hypothesized links between the latent variables and their observed indicators. The construct reliability of the latent variables was assessed using composite reliability values, calculated from the squared sum of the standardized factor loading divided by the squared sum of the standardized factor loading and the error variance terms (Hair, Black, Babin, \& Anderson, 2010). Second, we examined the structural model testing the direct and indirect links, via acceptance and denial coping, of the caregiving burden on the parents' QoL. Following recent recommendations (Preacher \& Hayes, 2008), the statistical significance of the indirect effects was estimated using bootstrap resampling procedures with 2000 samples and a $95 \%$ 
bias-corrected confidence interval (BC 95\% Cl). Finally, to ascertain that the parameters tested in the mediation model were valid for the asthma severity, the children's age and the family socioeconomic groups, we examined the baseline model for each group separately, and we subsequently conducted multi-group analyses testing the model's invariance between the groups. As suggested by Little (2013), we first tested the invariance on the associations between the latent variables and their observed indicators; once the measurement invariance was established, we examined the structural invariance, i.e., the invariance on the strength of the associations among the latent variables. The chisquare difference $\left(\Delta \chi^{2}\right)$ method was used to compare the unconstrained model with the models in which the measurement weights and the structural weights were fixed to be equal across groups.

\section{Results}

Sample characteristics

The sample was composed of 182 parents who assumed the role of primary caregivers of 112 children with asthma aged $8-12$ years $(M=10.23, S D=1.27 ; 69.6 \%$ male) and 70 adolescents with asthma aged $13-18$ years $(M=14.7 \mid, S D=1.49 ; 55.7 \%$ male $)$. The parents included 153 mothers (84.1\%) and 29 fathers (I5.9\%), between 27 and $7 \mathrm{I}$ years of age $(M=4 \mathrm{I} .06 ; S D=5.85)$. The majority of the parents were married or were living with a partner $(n=145,79.7 \%)$ and had low $(n=108,59.3 \%)$ or medium $(n=63,34.6 \%)$ SES, while 37 families $(20.3 \%)$ lived in single-parent households (i.e., they were single, divorced or widowed) and only II families (6\%) had high SES.

Regarding the children's clinical characteristics, 95 children (52.2\%) had intermittent asthma, 52 children (28.6\%) had mild persistent asthma, 29 children (I5.9\%) had moderate persistent asthma and 6 children $(3.6 \%)$ had severe persistent asthma. Thirty-seven children (20.3\%) also had comorbidities with other allergic diseases, such as allergic rhinitis $(n=13,7.1 \%)$, atopic dermatitis/eczema $(n=8,4.4 \%)$ and hypersensitivity to inhalant or food allergens $(n=16,8.8 \%)$. The average length of time since asthma diagnosis was 7.37 years $(S D=3.93)$ and most children were taking regular or emergency medication $(n=178,97.8 \%)$.

Descriptive statistics and multivariate analyses of covariance

Preliminary correlation analyses indicated that a greater use of denial as a coping strategy was significantly associated with parents' female gender $(r=.19, p=.01)$ and with the absence of comorbidities with other allergic diseases in the child $(r=-.15, p=.04)$. In addition, living in singleparent households was negatively associated with the parents' $Q \circ \mathrm{L}$ in the psychological $(r=-.18, p=$ 
$.02)$ and social domains $(r=-.18, p=.01)$. Accordingly, these variables were included as covariates in the multivariate analyses of covariance when appropriate. No significant associations were found for parents' age, children's gender and length of time since asthma diagnosis.

The descriptive statistics for the caregiving burden dimensions, coping and parents' QoL are presented in Table I. Regarding the caregiving burden, we found significant multivariate main effects of asthma severity, Wilks' $\lambda=.94 ; F_{(3,172)}=3.79, p=.01 ; \eta_{p}^{2}=.06$, children's age group, Wilks' $\lambda=$ $.94 ; F_{(3,172)}=3.38, p=.02 ; \eta_{p}{ }^{2}=.06$, and SES, Wilks' $\lambda=.90 ; F_{(3,172)}=6.29, p<.01 ; \eta_{p}{ }^{2}=.10$. The univariate analyses (Table I) showed higher levels of caregiving burden for parents of children with intermittent asthma when compared with parents of children with persistent asthma, but only on the relationship dimension. In addition, the parents of younger children presented higher levels of objective and subjective burden than parents of adolescents. Finally, parents with low SES had higher levels of relationship and subjective burden, than parents with medium/high SES. No significant interaction effects between asthma severity, age and SES group were found.

In addition, we found multivariate main effects of SES on coping, while controlling for parents' gender and comorbidities with other allergic diseases, Wilks' $\lambda=.96 ; F_{(2,171)}=3.43, p=.04$; $\eta_{\mathrm{P}}^{2}=.04$, and on parents' QoL, while controlling for type of household, Wilks' $\lambda=.92 ; F_{(4,170)}=3.97$, $p<.01 ; \eta_{\mathrm{p}}^{2}=.09$. The univariate analyses revealed that parents with low SES tended to use denial as a coping strategy more often and reported a poorer QoL on the psychological and environment domains than parents with medium/high SES (Table I). No multivariate main effects of asthma severity levels or children's age group or interaction effects between the factors were found for coping strategies and parents' QoL.

\section{The measurement model}

The measurement model testing the hypothesized links between the latent variables (caregiving burden, acceptance, denial and QoL) and their observed indicators had a good fit, with $\chi^{2}(38)=59.68, p=.0 \mathrm{I} ; \mathrm{CFI}=.97 ; \mathrm{RMSEA}=.06(p=.33 ; 90 \% \mathrm{Cl}=.03 / .08) ;$ and SRMR $=.05$. All observed indicators presented good factorial validity: standardized regression weights were above the threshold of .50 and statistically significant (Table 2$)$. The squared multiple correlations $\left(R_{\text {smc }}{ }^{2}\right)$ between each observed indicator and all other observed indicators were lower than .90, and the tolerance values $\left(I-R_{s m c}{ }^{2}\right)$ were higher than .10, indicating that each variable explained a substantial proportion of the total variance, and, thus, the model was not limited by multicollinearity problems (Kline, 2005). In addition, the construct reliability of the latent variables was good for caregiving burden and parents' QoL, with composite reliability values above the threshold of .70, and satisfactory for the acceptance and denial coping strategies, with composite reliability values near .60 . 
Table I | Descriptive statistics and univariate analyses of covariance by asthma severity, children's age and SES groups

\begin{tabular}{|c|c|c|c|c|c|c|c|c|c|}
\hline \multirow[b]{3}{*}{ Variable } & \multicolumn{2}{|c|}{ Asthma severity } & & \multicolumn{2}{|c|}{ Age group } & \multicolumn{4}{|c|}{ Socio-economic status } \\
\hline & $\begin{array}{l}\text { Intermittent } \\
\qquad(n=95)\end{array}$ & $\begin{array}{c}\text { Persistent } \\
(n=87)\end{array}$ & & $\begin{array}{l}\text { Children } \\
(n=1 \mid 2)\end{array}$ & $\begin{array}{l}\text { Adolescents } \\
(n=70)\end{array}$ & & $\begin{array}{c}\text { Low } \\
(n=108)\end{array}$ & $\begin{array}{l}\text { Medium/high } \\
\quad(n=74)\end{array}$ & \\
\hline & $M(S D)$ & $M(S D)$ & $F_{(1,172)}$ & $M(S D)$ & $M(S D)$ & $F_{(1,172)}$ & $M(S D)$ & $M(S D)$ & $F_{(1,172)}$ \\
\hline \multicolumn{10}{|l|}{ Caregiving burden } \\
\hline Relationship & $\mathrm{I} .64(0.80)$ & $\mathrm{I} .40(0.57)$ & $5.76^{*}$ & $1.57(0.68)$ & $\mathrm{I} .44(0.76)$ & 3.51 & I.6I (0.79) & $1.39(0.55)$ & $5.91^{*}$ \\
\hline Objective & $\mathrm{I} .82(0.8 \mathrm{I})$ & $\mathrm{I} .80(0.77)$ & 0.06 & $1.94(0.81)$ & $\mathrm{I} .60(0.7 \mathrm{I})$ & $9.73^{* *}$ & $\mathrm{I} .83(0.79)$ & $1.79(0.79)$ & 0.74 \\
\hline Subjective & $2.11(0.92)$ & $1.99(0.85)$ & 1.10 & $2.14(0.90)$ & $1.92(0.85)$ & $5.29^{*}$ & $2.21(0.97)$ & $1.83(0.69)$ & $10.79^{\text {** }}$ \\
\hline \multicolumn{10}{|l|}{ Coping strategies } \\
\hline Acceptance & $1.97(0.65)$ & $2.08(0.70)$ & 0.45 & $2.01(0.61)$ & $2.04(0.77)$ & 0.11 & $2.0 I(0.7 I)$ & $2.04(0.63)$ & 0.03 \\
\hline Denial & $0.85(0.70)$ & $0.84(0.79)$ & 0.37 & $0.88(0.74)$ & $0.79(0.75)$ & 1.15 & $0.97(0.8 \mathrm{I})$ & $0.67(0.60)$ & $6.67^{*}$ \\
\hline \multicolumn{10}{|l|}{ Quality of life } \\
\hline Physical & $3.89(0.61)$ & $3.90(0.57)$ & 0.03 & $3.86(0.58)$ & $3.95(0.6 \mathrm{I})$ & 1.94 & $3.86(0.63)$ & $3.96(0.5 \mathrm{I})$ & 2.28 \\
\hline Psychological & $3.83(0.58)$ & $3.90(0.62)$ & 0.82 & $3.86(0.58)$ & $3.87(0.63)$ & 0.50 & $3.80(0.64)$ & $3.95(0.52)$ & $4.79^{*}$ \\
\hline Social relationships & $3.86(0.63)$ & $4.03(0.69)$ & 3.14 & $3.91(0.64)$ & $3.99(0.70)$ & 1.13 & $3.89(0.73)$ & $4.02(0.55)$ & 2.75 \\
\hline Environment & $3.61(0.57)$ & $3.62(0.50)$ & 0.03 & $3.60(0.54)$ & $3.65(0.53)$ & 0.98 & $3.49(0.55)$ & $3.80(0.46)$ & $15.37^{* *}$ \\
\hline
\end{tabular}

${ }^{* *} p<.01 ;{ }^{*} p<.05$, two-tailed. 
Weak to moderate associations were found between the latent variables: caregiving burden was positively correlated with denial and negatively correlated with acceptance and with parents' QoL; acceptance and denial coping strategies were negatively associated with each other, and they were associated with parents' QoL, with positive correlations found between acceptance and QoL, and negative correlations found between denial and QoL.

The mediation model

The structural model testing the direct and indirect effects, via acceptance and denial coping strategies, of caregiving burden on parents' QoL (Figure I) had a good fit, with $\chi^{2}(39)=$ 63.88, $p=.0 \mathrm{I} ; \mathrm{CFI}=.96 ; \mathrm{RMSEA}=.06(p=.26 ; 90 \% \mathrm{Cl}=.03 / .09)$; and SRMR $=.06$, and explained 30\% of parents' QoL. Significant direct effects of caregiving burden were found on coping strategies $(\beta=-.27, p=.02$ for acceptance; $\beta=.40, p<.01$ for denial). In turn, acceptance and denial had significant direct effects on parents' QoL, with $\beta=.29, p=.0 \mathrm{I}$; and $\beta$ $=-.32, p=.0 \mathrm{I}$, respectively. No direct effect of caregiving burden on parents' $\mathrm{Q}$ oL was found $(\beta$ $=-.16, p=.10)$, but the indirect effects via coping strategies were statistically significant $(\beta=$ $.20, p<.01 ; \mathrm{BC} 95 \% \mathrm{Cl}=-.40 /-.09)$. We also examined the simple mediation models for each coping strategy, and both acceptance $(\beta=-.08, p=.04, \mathrm{BC} 95 \% \mathrm{Cl}=-.24 /-.02)$ and denial $(\beta=$ $.14, p<.01 ; \mathrm{BC} 95 \% \mathrm{Cl}=-.32 /-.05)$ emerged as significant mediators of the association between caregiving burden and parents' QoL.

\section{Multi-group analyses}

Table 3 presents the summary of fit statistics for the mediation model tested separately for the asthma severity groups (intermittent vs. persistent), the children's age groups (children aged 8-12 vs. adolescents aged 13-18) and the SES groups (low vs. medium/high), as well as the results from multi-group analyses comparing the unconstrained model with the nested models in which factor loadings and structural weights were sequentially and cumulatively fixed to be equal across groups. Regarding measurement invariance, the factor loadings of the observed indicators on their respective latent variables did not significantly differ between parents of children with intermittent asthma and parents of children with persistent asthma $\left(\Delta \chi^{2}(7)=6.25, p=.5 \mathrm{I}\right)$, between parents of children and parents of adolescents $\left(\Delta \chi^{2}(7)=9.06, p=.25\right)$, or between families with low SES and families with medium/high SES $\left(\Delta \chi^{2}(7)=4.22, p=.75\right)$. Assuming that the measurement model is invariant across groups, the results from the multi-group analyses also yielded no significant differences in the structural weights across asthma severity $\left(\Delta \chi^{2}{ }_{(5)}=\right.$ I.40, $p=.92)$, children's age $\left(\Delta \chi^{2}(5)=4.37, p=.50\right)$ or family SES $\left(\Delta \chi^{2}(5)=9.10, p=.1 \mathrm{I}\right)$ groups. 
Table 2 | Inter-correlations, factor loadings and reliability for observed indicators and latent variables

\begin{tabular}{|c|c|c|c|c|c|c|c|c|c|c|c|c|c|c|c|c|}
\hline \multirow[b]{2}{*}{ Variable } & \multicolumn{3}{|c|}{ Caregiving burden } & \multicolumn{3}{|c|}{ Acceptance } & \multicolumn{3}{|c|}{ Denial } & \multicolumn{4}{|c|}{ Parents' QoL } & \multirow[t]{2}{*}{$I-R_{s m c}{ }^{2}$} & \multirow{2}{*}{$\begin{array}{l}\text { Factor } \\
\text { loadings }\end{array}$} & \multirow{2}{*}{$\begin{array}{c}\text { Reliability } \\
a\end{array}$} \\
\hline & $\mathrm{I}$ & 2 & 3 & $\mathrm{~B}$ & $\overline{4}$ & 5 & $\mathrm{C}$ & 6 & 7 & $\mathrm{D}$ & 8 & 9 & 10 & & & \\
\hline A. Caregiving burden & & & & $-.26^{* *}$ & & & $.39^{* *}$ & & & $-.36^{* *}$ & & & & & & .85 \\
\hline I. Relationship & - & & & & & & & & & & & & & .33 & $.82^{* *}$ & .86 \\
\hline 2. Objective & $.72^{* *}$ & - & & & & & & & & & & & & .23 & $.88^{* *}$ & .88 \\
\hline 3. Subjective & $.57^{* *}$ & $.67^{* *}$ & - & & & & & & & & & & & .45 & $.74^{* *}$ & .85 \\
\hline B. Acceptance & & & & & & & $-.34^{* * *}$ & & & $.40^{* * *}$ & & & & & & .63 \\
\hline 4. Acceptance I & $-.20^{* *}$ & -.08 & -.14 & & - & & & & & & & & & .61 & $.62^{* *}$ & - \\
\hline 5. Acceptance 2 & $-.25^{* *}$ & -.12 & -.12 & & $.46^{* *}$ & - & & & & & & & & .46 & $.74^{* *}$ & - \\
\hline C. Denial & & & & & & & & & & $-.46^{* *}$ & & & & & & .58 \\
\hline 6. Denial I & $.25^{* *}$ & $.20^{* *}$ & $.28^{* *}$ & & -.12 & -.14 & & - & & & & & & .56 & $.66^{* *}$ & - \\
\hline 7. Denial 2 & $.25^{* *}$ & .14 & $.15^{*}$ & & $-.17^{*}$ & $-.18^{*}$ & & $.4 I^{* *}$ & - & & & & & .61 & $.62^{* *}$ & - \\
\hline D. Parents' QoL & & & & & & & & & & & & & & & & .85 \\
\hline 8. Physical & $-.28^{* *}$ & $-.18^{*}$ & $-.29^{* *}$ & & $.19^{*}$ & $.23^{* *}$ & & $-.16^{*}$ & $-.25^{* *}$ & & - & & & .57 & $.66^{* *}$ & .81 \\
\hline 9. Psychological & $-.28^{* *}$ & $-.20^{* *}$ & $-.35^{* *}$ & & $.21^{* *}$ & $.23^{* *}$ & & $-.24^{* *}$ & $-.24^{* *}$ & & $.58^{* *}$ & - & & .24 & $.87^{* *}$ & .83 \\
\hline 10. Social & $-.23^{* *}$ & -.11 & $-.19^{* *}$ & & $.22^{* *}$ & $.28^{* *}$ & & $-.22^{* *}$ & $-.18^{*}$ & & $.42^{* *}$ & $.68^{* *}$ & - & .44 & $.75^{* *}$ & .73 \\
\hline II. Environmental & $-.29^{* *}$ & $-.21^{* *}$ & $-.37^{* *}$ & & $.15^{*}$ & $.20^{* *}$ & & $-.30^{* *}$ & $-.24^{* *}$ & & $.54^{* *}$ & $.63^{* *}$ & $.56^{* *}$ & .44 & $.75^{* *}$ & .82 \\
\hline
\end{tabular}

Note. Inter-correlations and composite reliability for latent variables are shown in boldface.

${ }^{a}$ Cronbach's $\alpha$ values were calculated for observed indicators and composite reliability values were computed for latent variables.

${ }^{* *} p<.01 ;{ }^{*} p<.05$, two-tailed. 


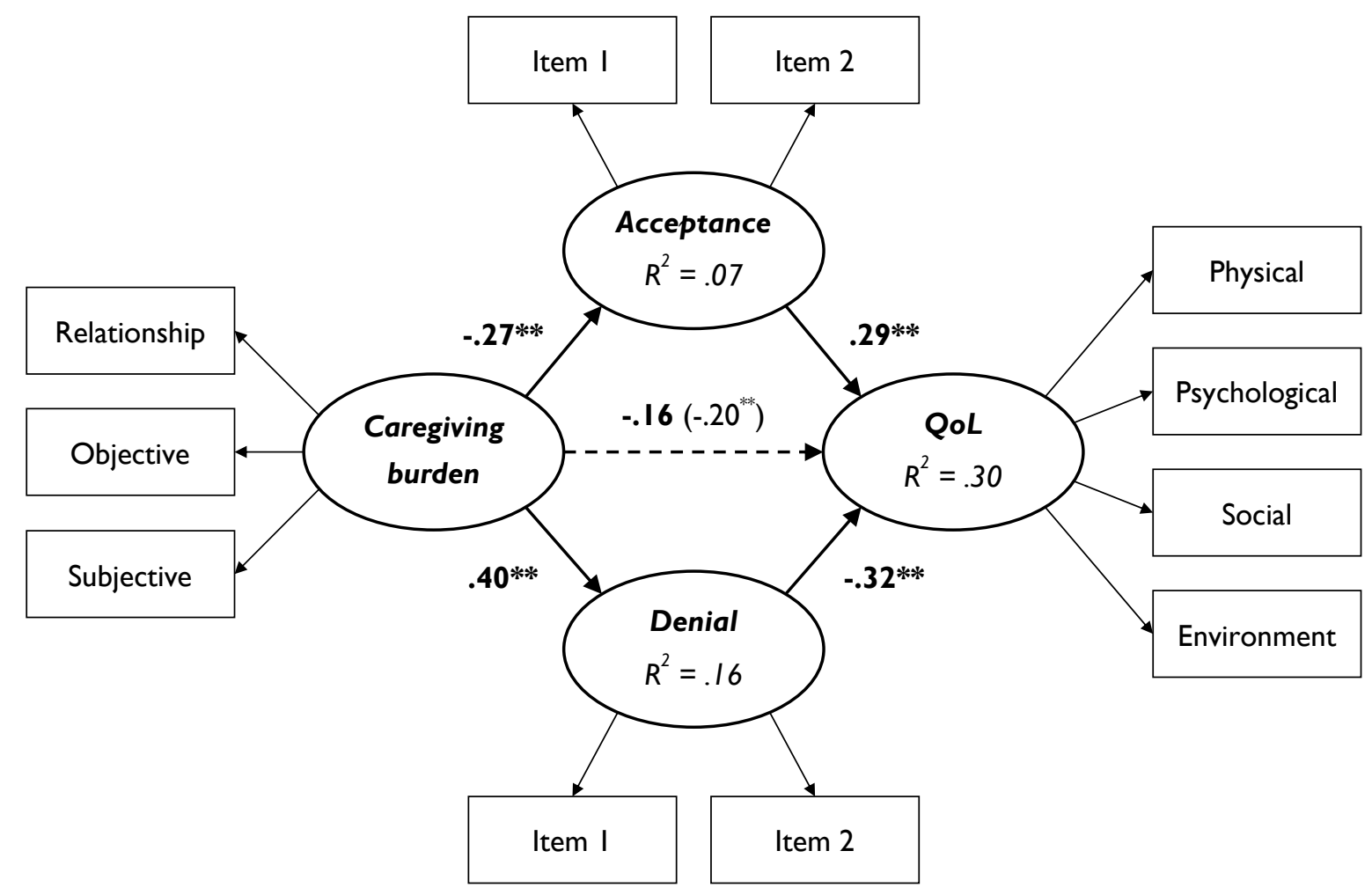

Figure I | SEM testing the direct and indirect effects, via acceptance and denial coping strategies, of caregiving burden on parents' QoL

Note. Bold figures represent the standardized regression weights for the direct paths; non-bold figures in brackets represent the standardized regression weights for the indirect path. For simplicity, measurement error terms are not shown.

** $p \leq .01 ;{ }^{*} p \leq .05$.

Table 3 | Multi-group analyses testing measurement and structural invariance across asthma severity, children's age and SES groups

\begin{tabular}{|c|c|c|c|c|c|c|c|c|}
\hline & $\chi^{2}$ & $d f$ & $\mathrm{CFI}$ & $\begin{array}{c}\text { RMSEA } \\
{[90 \% \mathrm{Cl}]}\end{array}$ & SRMR & $\Delta \chi^{2}$ & $\Delta d f$ & $\Delta \mathrm{CFI}$ \\
\hline \multicolumn{9}{|l|}{ Asthma severity groups } \\
\hline \multicolumn{9}{|l|}{ Summary of fit statistics } \\
\hline Intermittent & 53.70 & 39 & .97 & $.06[.00 / .10]$ & .06 & & & \\
\hline Persistent & 40.72 & 39 & .99 & $.02[.00 / .08]$ & .08 & & & \\
\hline \multicolumn{9}{|l|}{ Multi-group analyses } \\
\hline Unconstrained model & 94.42 & 78 & .98 & $.03[.00 / .06]$ & .06 & - & - & - \\
\hline Measurement weights & 100.67 & 85 & .98 & $.03[.00 / .05]$ & .06 & 6.25 & 7 & $<.01$ \\
\hline Structural weights & 102.07 & 90 & .98 & $.03[.00 / .05]$ & .06 & $\mathrm{I} .40$ & 5 & $<.01$ \\
\hline \multicolumn{9}{|l|}{ Children's age groups } \\
\hline \multicolumn{9}{|l|}{ Summary of fit statistics } \\
\hline Children 8-12 & $58.33^{*}$ & 39 & .95 & $.07[.03 / .10]$ & .06 & & & \\
\hline Adolescents 13-18 & $66.47^{* *}$ & 39 & .91 & $.10[.06 / .14]^{*}$ & .08 & & & \\
\hline \multicolumn{9}{|l|}{ Multi-group analyses } \\
\hline Unconstrained model & $124.90^{* *}$ & 78 & .93 & $.06[.04 / .08]$ & .06 & - & - & - \\
\hline
\end{tabular}




$\begin{array}{lllllllll}\text { Measurement weights } & 133.96^{* *} & 85 & .93 & .06[.04 / .07] & .07 & 9.06 & 7 & <.01 \\ \text { Structural weights } & 138.33^{* *} & 90 & .93 & .06[.04 / .07] & .07 & 4.37 & 5 & <.01\end{array}$

\begin{tabular}{|c|c|c|c|c|c|c|c|c|}
\hline \multicolumn{9}{|l|}{ SES groups } \\
\hline \multicolumn{9}{|l|}{ Summary of fit statistics } \\
\hline Low & $60.29^{*}$ & 39 & .95 & $.07[.03 / .1 \mathrm{I}]$ & .07 & & & \\
\hline Medium/high & 51.08 & 39 & .95 & $.07[.00 / .1 \mathrm{I}]$ & .08 & & & \\
\hline \multicolumn{9}{|l|}{ Multi-group analyses } \\
\hline Unconstrained model & $111.39^{* *}$ & 78 & .95 & $.05[.03 / .07]$ & .07 & - & - & - \\
\hline Measurement weights & $\left.|| 5.6\right|^{*}$ & 85 & .96 & $.05[.02 / .06]$ & .07 & 4.22 & 7 & .01 \\
\hline Structural weights & $|24.7|^{* *}$ & 90 & .95 & $.05[.02 / .07]$ & .09 & 9.10 & 5 & .01 \\
\hline
\end{tabular}

${ }^{* *} p \leq .01 ;{ }^{*} p \leq .05$

\section{Discussion}

The main contribution of the present study was the examination of a mediational hypothesis based on a solid theoretical background, namely the multi-dimensional model of the caregiving process and the stress and coping theory. The findings allowed the ascertainment of the caregiving burden as an important risk factor for parent's QoL impairments, as well as the identification of the mediating role of specific coping strategies, namely, a greater use of acceptance and a lower use of denial, for positive adaptation outcomes, which remain fundamental to research and clinical practice.

Our first set of results showed that parents of children with intermittent asthma, parents of younger children and parents with low SES were at greater risk of experiencing higher levels of caregiving burden. Although higher levels of caregiving burden reported by parents of children with intermittent asthma when compared with parents of children with persistent asthma were somewhat unexpected, they were significant only for the relationship burden domain. These findings suggest that, when asthma does not inflict severe limitations on the child's functioning, the parents may be more prone to interpret their child's demands for care and attention as over and above the needs warranted by the pediatric health condition. Furthermore, the asthma management routines may be less rigid in the daily functioning of families of children with intermittent asthma and, consequently, imply specific contingencies in therapeutic monitoring and adherence. For instance, decision-making about avoiding environmental triggers of asthma exacerbations (e.g., going out or meet up with friends) or using emergency medication can cause additional parent-child conflicts, which are eventually reflected in higher levels of relationship burden. Even if this explanatory hypothesis requires further research, our results strengthen the importance of addressing caregiving burden as a multi-dimensional construct (Savundranayagam 
et al., 20II), because clinical asthma characteristics may have a differential impact on the specific dimensions of parental burden.

Regarding the children's age groups, the higher levels of caregiving burden found for parents of children in comparison with parents of adolescents were consistent with our assumption that younger children could be more dependent on parental care and less able to communicate their healthcare needs, thus increasing the parents' responsibilities and worries. Similarly, Floyd and Gallagher (1997) described greater time demands in providing care to children with chronic illnesses or disabilities in mothers of children aged 6-12 years compared with mothers of adolescents aged 13 years and older. Along with the functional limitations related to the health condition, normative developmental capabilities may also influence the caregiving process. Therefore, conceptual models of adaptation to pediatric health conditions could be improved through the inclusion of child's age-related characteristics as important factors associated with the caregivers' outcomes.

Higher levels of caregiving burden and lower QoL were also found for parents with low SES when compared with parents with medium/high SES. These results were consistent with our hypothesis and support previous findings showing that the opportunity to afford additional support from healthcare service providers and to successfully balance work-caregiving responsibilities may ease caregiving demands and contribute to positive adaptation outcomes in parents (Gates \& Akabas, 2012). Moreover, parents with low SES were more likely to use denial as a coping strategy suggesting that when parents have insufficient external resources to help them cope with the stressful events, avoidant strategies are preferred. These results should be interpreted within the Portuguese current economic context and healthcare system, because additional financial burdens may have inflated the levels of caregiving burden and, consequently, contributed to poorer QoL outcomes.

Caregiving burden was negatively associated with parents' QoL, but only indirectly via a greater use of denial and a lower use of acceptance as coping strategies, confirming our second hypothesis. The absence of direct associations between the caregiving burden and the parents' adaptation outcomes has previously been observed in other studies (e.g., King et al., 1999), and emphasizes the important role of stress processing mechanisms for the well-being of parents caring for a child with a chronic condition (Raina et al., 2004; Wallander et al., 1989). The parents' perceptions of increased burden and negative affect related to caregiving may hinder the use of acceptance coping and predispose them to deny or minimize stressful events, either related or unrelated to parental caregiving. The reduced use of acceptance as a coping strategy may also reflect the parents' difficulties in accommodating and reconciling the caregiving demands with their individual goals and expectations (Pakenham, 2008). In turn, and as expected, these coping tendencies were negatively linked to the parents' QoL, which is basically 
conceptualized in relation to individual goals, expectations, standards and concerns (The WHOQOL Group, 1998).

These results were strengthened by the ascertainment of the model's invariance across asthma severity, children's age and SES groups. Although parents of children with intermittent asthma, young age and low SES presented higher levels of caregiving burden, coping dispositions marked by greater use of acceptance and less use of avoidance strategies have operated as resource factors positively linked to parents' QoL outcomes, regardless of the sociodemographic and clinical conditions. The acceptance coping mechanisms underlying the adaptation outcomes for parents caring for a child with asthma are similar to those described for normative parenting (Duncan, Coatsworth, \& Greenberg, 2009), which suggests a general applicability of mindful parenting models and acceptance-based interventions in the context of pediatric asthma.

\section{Limitations of the study}

The results from our study should be interpreted with caution due to some limitations in the study design and procedures. First, even though the directional paths tested in the mediation analyses have been hypothesized according to widely accepted theoretical models, the study's cross-sectional design prevents the establishment of causal relationships among the variables. Second, the required sample size for SEM has been suggested to vary between a minimum of 100-200 individuals, but more complex models (i.e., with higher number of distinct parameters to be estimated) may require larger sample sizes in order to achieve the necessary power to detect small effects (Kline, 2005). Third, the non-probabilistic sample collection method may have biased the distribution of clinical (e.g., asthma severity levels) and sociodemographic characteristics (e.g., family SES and parents' gender) of the sample and may limit the generalizability of the results. The low percentage of parents of children classified by persistent levels of asthma severity, although consistent with the distribution of asthma severity levels typically observed in the Portuguese pediatric population (Gaspar, Almeida, \& Nunes, 2006), required the dichotomization of the asthma severity variable, which increased the intragroup variability and may have overlooked potential group differences. In addition, the classification of asthma severity levels by the child's physician was based on existing guidelines at the time of sample collection (GINA, 2008), and they do not represent the current recommendation of using both the parents' and the child's information for the assessment of asthma symptom control (GINA, 20I4). Similarly, our sample was mainly composed of mothers with low/medium SES, which precluded the examination of gender differences and required the dichotomization of the SES variable even if this distribution was consistent with the users' profile in Portuguese outpatient public healthcare services and with previous studies with family caregivers (e.g., 
Canning et al., 1996; Everhart et al., 2008). Finally, the reliability values in our sample for the coping variables (acceptance and denial) were below the threshold of .70, which should be recognized as a limitation of the study even if they were slightly higher than those reported in the original studies of the Brief-COPE instrument and are considered by the author to be acceptable since each scale consists of two items only (Carver, 1997).

Implications for clinical practice and research

Two main implications for clinical practice in pediatric asthma settings can be drawn from the present study. First, it is important that health professionals recognize that pediatric asthma affects not only the child but the family as a whole (Fiese et al., 2008) and that parents, particularly those caring for a younger child or with scarce socio-economic resources, may be at greater risk for experiencing higher levels of caregiving burden. Therefore, the caregiving burden should be routinely assessed and elected as a strategic intervention target for family-centered psychosocial interventions aimed at promoting parents' adaptation processes and outcomes. Second, coping tendencies characterized by greater use of acceptance and less use of denial can be encouraged in parents caring for a child with asthma, regardless of asthma severity or sociodemographic characteristics, in order to diminish the deleterious effects of the caregiving burden on the parents' QoL outcomes. Cognitive-behavioral therapies have been extensively recognized for their effectiveness in promoting more realistic appraisals of stressful events and the adequate use of coping resources in patients with health conditions (Ridder \& Schreurs, 200I). In the specific context of caring for a child with asthma, acceptance-based interventions are expected to be effective in reappraising the caregiving demands and parent-child relationships as less burdensome, in developing coping tendencies marked by acceptance as an alternative to experiential avoidance, and, ultimately, in improving parental adaptation. Specifically, mindfulness training is likely to decrease caregiving-related stress, through the exposure to negative emotional states and the broadening of attention to more varied aspects of the caregiving experience and ways of coping with it (Bazzano et al., 2015; Minor et al., 2006).

Further longitudinal research should be undertaken to clarify the direction of the direct and indirect pathways proposed in our mediation model, as well as to examine the role of awareness, distraction, prevention of negative emotions and constructive self-assertion as specific mindful coping strategies (Tharaldsen, Bru, \& Wilhelmsen, 20II) mediating the caregiving process. Besides, the efficacy of acceptance and mindful-based interventions to promote parental adaptation to pediatric asthma and the benefits of its availability as a public resource in pediatric healthcare services can only be ascertained by longitudinal research, namely, randomized controlled clinical trials and cost-effectiveness studies. 


\section{References}

Bazzano, A., Wolfe, C., Zylowska, L., Wang, S., Schuster, E., Barrett, C., \& Lehrer, D. (20I5). Mindfulness Based Stress Reduction (MBSR) for parents and caregivers of individuals with developmental disabilities: A community-based approach. Journal of Child and Family Studies, 24, 298-308. doi:10.1007/s 10826-013-9836-9

Benn, R., Akiva, T., Arel, S., \& Roeser, R. W. (20I2). Mindfulness training effects for parents and educators of children with special needs. Developmental Psychology, 48, I476-1487. doi: $10.1037 / \mathrm{a} 0027537$

Blount, R., Simons, L., Devine, K., Jaaniste, T., Cohen, L., Chambers, C., \& Hayutin, L. (2008). Evidence-based assessment of coping and stress in pediatric psychology. Journal of Pediatric Psychology, 33, I02I-1045. doi:10.1093/jpepsy/jsm07I

Browne, M., \& Cudeck, R. (1993). Alternative ways of assessing model fit. In K. Bollen, \& J. Long (Eds.), Testing structural equation models (Pp. 136-162). Newbury Park, CA: Sage.

Canning, R., Harris, E., \& Kelleher, K. (1996). Factors predicting distress among caregivers to children with chronic medical conditions. Journal of Pediatric Psychology, 21, 735-749. doi:10.1093/jpepsy/21.5.735

Carona, C., Crespo, C., \& Canavarro, M. C. (2013). Similarities amid the difference: Caregiving burden and adaptation outcomes in dyads of parents and their children with and without cerebral palsy. Research in Developmental Disabilities, 34, 882-893. doi:10.1016/j.ridd.2012.12.004

Carona, C., Pereira, M., Moreira, H., Silva, N., \& Canavarro, M. C. (20I3). The disability paradox revisited: Quality of life and family caregiving in pediatric cerebral palsy. Journal of Child and Family Studies, 22, 97I-986. doi:10.1007/s 10826-012-9659-0

Carver, C. (1997). You want to measure coping but your protocol's too long: Consider the Brief COPE. International Journal of Behavioral Medicine, 4, 92-100. doi:I0.1207/s I5327558ijbm040I_6

Carver, C., Scheier, M., \& Weintraub, J. (1989). Assessing coping strategies: A theoretically based approach. Journal of Personality and Social Psychology, 56(2), 267-283.

Crespo, C., Carona, C., Silva, N., Canavarro, M. C., \& Dattilio, F. (20I I). Understanding the quality of life for parents and their children who have asthma: Family resources and challenges. Contemporary Family Therapy, 33, I79-196. doi:10.1007/s 10591-01 I-9155-5

Duncan, L. G., Coatsworth, J. D., \& Greenberg, M. T. (2009). A model of mindful parenting: Implications for parent-child relationships and prevention research. Clinical Child and Family Psychology Review, 12, 255-270. doi:10.1007/s 10567-009-0046-3

Everhart, R., Fiese, B. H., \& Smyth, J. (2008). A cumulative risk model predicting caregiver quality 
of life in pediatric asthma. Journal of Pediatric Psychology, 33, 809-8I8. doi:10.1093/jpepsy/jsn028

Fiese, B. H., Wamboldt, F., \& Anbar, R. (2005). Family asthma management routines: Connections to medical adherence and quality of life. Journal of Pediatrics, 146, 17I-I76. doi:10.1016/j.jpeds.2004.08.083

Fiese, B. H., Winter, M., Anbar, R., Howell, K., \& Poltrock, S. (2008). Family climate of routine asthma care: Associating perceived burden and mother-child interaction patterns to child well-being. Family Process, 47, 63-79. doi:10.1 I I I/j.1545-5300.2008.00239.x

Floyd, F., \& Gallagher, E. (1997). Parental stress, care demands, and use of support services for school-age children with disabilities and behavior problems. Family Relations, 46(4), 359371

Folkman, S., \& Lazarus, R. (1980). An analysis of coping in a middle-aged community sample. Journal of Health \& Social Behavior, 2 I (3), 219-239.

García-Alberca, J. M., Cruz, B., Lara, J. P., Garrido, V., Gris, E., Lara, A., \& Castilla, C. (20/2). Disengagement coping partially mediates the relationship between caregiver burden and anxiety and depression in caregivers of people with Alzheimer's disease. Results from the MÁLAGA-AD study. Journal of Affective Disorders, 136, 848-856. doi:10.1016/j.jad.2011.09.026

Gaspar, A., Almeida, M., \& Nunes, C. (2006). Epidemiologia da asma grave [Epidemiology of severe asthma]. Revista Portuguesa de Imunoalergologia, I4(S2), 27-4I.

Gates, L., \& Akabas, S. (20I2). Meeting the demands of work and responsibilities of caring for a child with asthma: Consequences for caregiver well-being. Journal of Social Service Research, 38, 656-67I. doi:I0.1080/0I488376.2012.70948।

Gau, B. S., Chen, Y. C., Lo, L. H., Chang, M., Chao, Y. M., Chiang, B. L., \& Yao, G. (2010). Clinical applicability of the World Health Organization Quality of Life Scale Brief Version (WHOQOL-BREF) to mothers of children with asthma in Taiwan. Journal of Clinical Nursing, 19, 8I I-819. doi:10.1 I I //j.1365-2702.2009.02934.x

Global Initiative for Asthma. (2008). Global strategy for asthma management and prevention Updated 2008. Retrieved from http://www.ginasthma.org

Global Initiative for Asthma. (2014). Global strategy for asthma management and prevention Revised 20/4. Retrieved from http://www.ginasthma.org

Hair, J., Black, W., Babin, B., \& Anderson, R. (2010). Multivariate data analysis (7th ed.). New Jersey, NJ: Prentice Hall.

Halterman, J., Yoos, H., Conn, K., Callahan, P., Montes, G., Neely, T., \& Szilagyi, P. (2004).The impact of childhood asthma on parental quality of life. Journal of Asthma, 4I, 645-653. doi: 10.108I/JAS-200026410 
Hatzmann, J., Heymans, H., Ferrer-i-Carbonell, A., van Praag, B., \& Grootenhuis, M. (2008). Hidden consequences of success in pediatrics: Parental health-related quality of life - Results from the Care Project. Pediatrics, I22, 1030-1038. doi:10.1542/peds.2008-0582

Hayes, S. C. (2004). Acceptance and Commitment Therapy, Relational Frame Theory, and the third wave of behavior therapy. Behavior Therapy, 35, 639-665. doi:10.1016/500057894(04)80013-3

Hu, L., \& Bentler, P. (1999). Cutoff criteria for fit indexes in covariance structure analysis: Conventional criteria versus new alternatives. Structural Equation Modeling: A Multidisciplinary Journal, 6, I-55. doi:10.1080/10705519909540 I I8

Kabat-Zinn, J. (1990). Full catastrophe living: Using the wisdom of your body and mind to face stress, pain, and illness. New York, NY: Bantam Doubleday Dell.

King, G., King, S., Rosenbaum, P., \& Goffin, R. (1999). Family-centered caregiving and well-being of parents of children with disabilities: Linking process with outcome. Journal of Pediatric Psychology, 24, 4I-53. doi:10.1093/jpepsy/24.I.4I

Kline, R. B. (2005). Principles and practice of structural equation modeling (2nd ed.). New York, NY: Guilford.

Larson, E. (2010). Psychological well-being and meaning-making when caregiving for children with disabilities: Growth through difficult times or sinking inward. OTJR: Occupation, Participation and Health, 30, 78-86. doi: 10.3928/I5394492-20100325-03

Lazarus, R., \& Folkman, S. ( 1984). Stress, appraisal, and coping. New York, NY: Springer.

Little, T. (Ed.). (2013). The Oxford handbook of quantitative methods in psychology (Vol. I). New York, NY: Oxford University Press.

Livneh, H. (2009). Denial of chronic illness and disability: Part I. Theoretical, functional, and dynamic perspectives. Rehabilitation Counseling Bulletin, 52, 225-236. doi: $10.1177 / 0034355209333689$

Minor, H., Carlson, L., Mackenzie, M., Zernicke, K., \& Jones, L. (2006). Evaluation of a MindfulnessBased Stress Reduction (MBSR) program for caregivers of children with chronic conditions. Social Work in Health Care, 43, 91-109. doi:I0.1300/J010v43n0I_06

Montgomery, R., \& Kosloski, K. (2006). The league of experienced family caregivers: Measure development. Milwaukee, WI: University of Wisconsin-Milwaukee.

Moos, R., \& Schaefer, J. (1993). Coping resources and processes: Current concepts and measures. In L. Goldberger \& S. Breznitz (Eds.), Handbook of stress: Theoretical and clinical aspects (2nd ed., Pp. 234-257). New York, NY: Free Press.

Pais-Ribeiro, J. L., \& Rodrigues, A. P. (2004). Questões acerca do coping: A propósito do estudo de adaptação do Brief Cope [Some questions about coping: The study of the Portuguese adaptation of the Brief COPE]. Psicologia, Saúde \& Doenças, 5(I), 3-I5. 
Pakenham, K. (2008). Making sense of caregiving for persons with multiple sclerosis (MS): The dimensional structure of sense making and relations with positive and negative adjustment. International Journal of Behavioral Medicine, 15, 24I-252. doi: $10.1080 / 10705500802222345$

Pearlin, L., \& Schooler, C. (1978). The structure of coping. Journal of Health \& Social Behavior, 19(1), 2-21.

Preacher, K., \& Hayes, A. (2008). Asymptotic and resampling strategies for assessing and comparing indirect effects in multiple mediator models. Behavior Research Methods, 40, 879-89|. doi:I0.3758/BRM.40.3.879

Raina, P., O’Donnell, M., Schwellnus, H., Rosenbaum, P., King, G., Brehaut, J., ... Wood, E. (2004). Caregiving process and caregiver burden: Conceptual models to guide research and practice. BioMed Central Pediatrics, 4, I. doi:I0.I I86/I47I-243I-4-I

Ridder, D., \& Schreurs, K. (200I). Developing interventions for chronically ill patients: Is coping a helpful concept? Clinical Psychology Reviews, 2I, 205-240. doi:10.1016/S02727358(99)00046-X

Roesch, S., \& Weiner, B. (200I). A meta-analytic review of coping with illness: Do causal attributions matter? Journal of Psychosomatic Research, 50, 205-219. doi:10.1016/500223999(0I)00I88-X

Savundranayagam, M., Montgomery, R., \& Kosloski, K. (20II). A dimensional analysis of caregiver burden among spouses and adult children. The Gerontologist, 5I, 32I-33I. doi:10.1093/geront/gnq 102

Silva, N., Carona, C., Crespo, C., \& Canavarro, M. C. (2015). Quality of life in pediatric asthma patients and their parents: A meta-analysis on 20 years of research. Expert Review of Pharmacoeconomics \& Outcomes Research. Advance online publication. doi: I0.1586/14737I67.20I5.1008459

Simões, M. (1994). Investigações no âmbito da aferição nacional do teste das Matrizes Progressivas de Raven [Raven's Progressive Matrices: Aferition studies]. (Unpublished doctoral dissertation). University of Coimbra, Coimbra, Portugal.

Tharaldsen, K. B., Bru, E., \& Wilhelmsen, I. (20II). Mindful coping and mental health among adolescents. The International Journal of Mental Health Promotion, 13, 21-31. doi: $10.1080 / 14623730.2011 .97 / 5653$

The WHOQOL Group. (1998). Development of the World Health Organization WHOQOLBREF quality of life assessment. Psychological Medicine, 28(3), 55I-558.

van Gent, R., van Essen, L., Rovers, M., Kimpen, J., van der Ent, C., \& de Meer, G. (2007). Quality of life in children with undiagnosed and diagnosed asthma. European Journal of Pediatrics, 166, 843-848. doi:10.1007/s00431-006-0358-y 
Vaz-Serra, A., Canavarro, M. C., Simões, M., Pereira, M., Gameiro, S., Quartilho, M. J., ... Paredes, T. (2006). Estudos psicométricos do instrumento de avaliação da qualidade de vida da Organização Mundial de Saúde (WHOQOL-Bref) para português de Portugal [Psychometric studies of the World Health Organization Quality of Life instrument (WHOQOL-Bref) in Portugal]. Psiquiatria Clínica, 27(2), 4I-49.

Wallander, J., Varni, J., Babani, L., Banis, H., DeHaan, C., \& Wilcox, K. (1989). Disability parameters, chronic strain, and adaptation of physically handicapped children and their mothers. Journal of Pediatric Psychology, 14, 23-42. doi:I0.1093/jpepsy//4.1.23

Whittingham, K., Wee, D., Sanders, M. R., \& Boyd, R. (20/3). Predictors of psychological adjustment, experienced parenting burden and chronic sorrow symptoms in parents of children with cerebral palsy. Child: Care, Health and Development, 39, 366-373. doi:10.1 III/j.1365-22I4.20I2.01396.x 